\title{
Recycler des masques chirurgicaux grâce aux accélérateurs de particules
}

Nicolas Delerue (delerue@lal.in2p3.fr)

Laboratoire de physique des 2 infinis - Irène Joliot-Curie (IJCLab), Université Paris-Sud, Bât. 100,

91405 Orsay Cedex

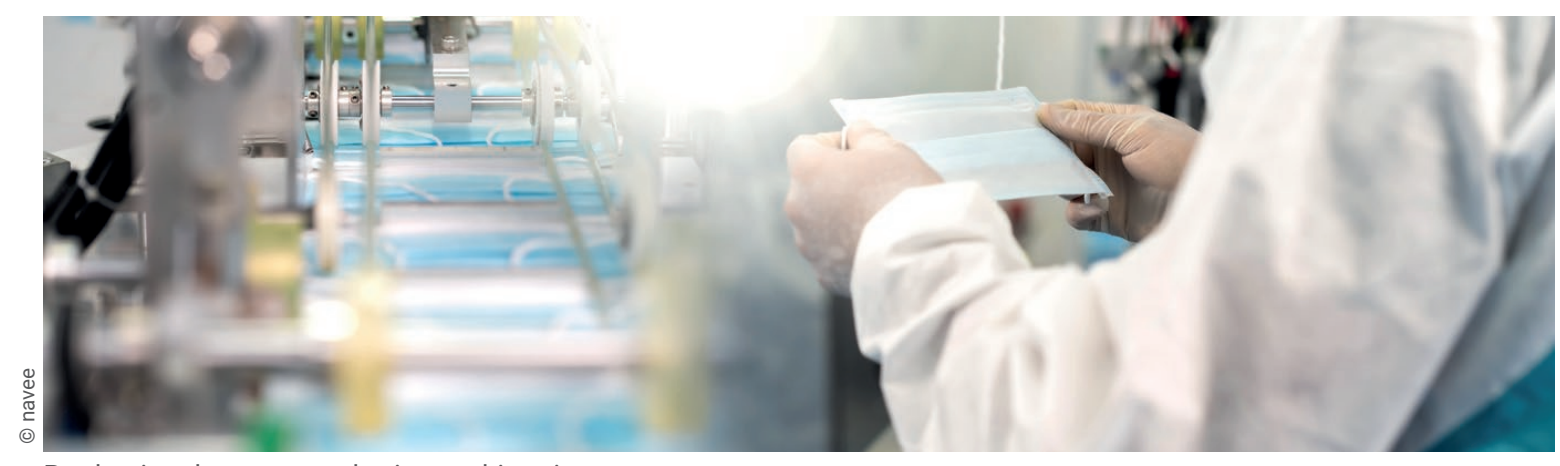

Production de masques de visage chirurgicaux

Au début de la propagation du SARS-CoV-2 en France, les personnels soignants ont été confrontés à une tension forte dans l'approvisionnement en matériel de protection et en particulier de masques. En conditions normales, un équipement de protection usagé ne doit pas être réutilisé. Cependant, des solutions de recyclage ont été étudiées à cause des difficultés d'approvisionnement [1]. L'une des solutions pour pallier à ces difficultés consiste en un lavage des masques (et des autres équipements de protection) à haute température avec détergent, suivi d'une stérilisation par rayons gamma ou bêta. Pendant le premier confinement, une collaboration associant le CHU de Grenoble, le CEA et le CNRS s'est mise en place pour étudier ces solutions de recyclage.

Une de ces solutions utilise le fait qu'un fort flux d'électrons (rayons bêta) ou de photons de haute énergie (rayons gamma) a un effet stérilisant important. Ce principe est exploité depuis de nombreuses années par des industriels français (Ionisos [2], ITHPP [3]) pour stériliser des équipements médicaux. Cette technique a l'avantage de permettre une stérilisation sans contact ni manipulation des équipements à travers leur emballage définitif. De nombreux instruments utilisés en chirurgie sont déjà stérilisés par cette technique, qui est d'ailleurs normalisée (norme ISO 11137 [4]). L'intensité du flux de particules et leur énergie doivent être choisies de manière à ce que la dose déposée garantisse une stérilisation complète des instruments, y compris dans les zones les moins accessibles ou les moins exposées, sans qu'il y ait un risque d'activation des matériaux.
De telles irradiations peuvent se faire avec des accélérateurs d'électrons compacts de basse énergie (une dizaine de $\mathrm{MeV}$ ), permettant de délivrer en quelques secondes une dose de plusieurs dizaines ou centaines de kilogray selon les besoins [3].

Les premières études ont montré qu'un tel traitement fait perdre aux masques chirurgicaux environ $2 \%$ de leur efficacité de filtration, permettant leur recyclage. Pour les masques FFP2, le traitement par rayons gamma semble dégrader les performances, mais une autre solution basée sur une exposition à l'oxyde d'éthylène $\mathrm{C}_{2} \mathrm{H}_{4} \mathrm{O}$ gazeux semble efficace [1].

Au niveau international, un groupe de travail s'est formé pour travailler sur cette problématique. Le 30 avril 2020,l'Agence Internationale pour l'Énergie Atomique (AIEA) a publié une note [5] se basant sur des travaux menés dans plusieurs pays, qui confirment l'utilité des rayonnements ionisants dans la stérilisation des équipements de protection individuels, mais mettent en garde contre le fait que ces rayonnements dégradent les filtres électrostatiques des masques FFP2. Pour ces derniers, il est donc préférable d'utiliser d'autres techniques de stérilisation.

\section{Références}

1 - Article de M. Grousson dans CNRS Le journal (9 avril 2020) : https://cutt.ly/masques-de-protection-recyclage

2. www.ionisos.com/nos-activites/steriliser/

3. https://cutt.ly/ithpp-alcen-sterilisation

4. www.iso.org/fr/standard/33952.html

5• https://cutt.ly/iaea-org-radiation-in-sterilizing 